\title{
La expresión corporal, como medio de comunicación educacional
}

\section{Corporal expression, as a means of educational communication}

MSc. Hugo Guerrero Laurido $\mathrm{PhD}$

Universidad de Especialidades Espíritu Santo, Ecuador

Autor para correspondencia: hguerrero@uees.edu.ec

Fecha de recepción: 31 de Julio de 2017 - Fecha de aceptación: 10 de Agosto de 2017

Resumen: La expresión corporal supone un contenido importante como un mecanismo de comunicación con su medio. Es decir, se trata de un contenido de origen multidisciplinar, por lo que debemos definir cuál es el concepto del que partimos, así como las corrientes o pilares que sustentan nuestro trabajo en el ámbito educativo. También vamos a definir las finalidades $\mathrm{u}$ objetivos que pretendemos alcanzar a través de las capacidades expresivas, secuenciando éstas y mostrando algunas nociones didácticas para su desarrollo. El concepto de expresión corporal se ha analizado desde diferentes vertientes o ámbitos, como afirma Ortiz en su texto sobre la expresión corporal (2002), aunque este término es ambiguo y polivalente, nosotros lo abordamos desde el ámbito pedagógico, como un instrumento educativo más; pero a la vez, nunca dejando de lado lo que toda conducta o manifestación comunica. Ortiz (2002:25) realiza una aproximación al concepto de expresión corporal a partir de numerosas aportaciones de diversos autores. A partir de estas afirma que en el ámbito de la educación física y cualquier otra materia la expresión corporal debe contribuir al desarrollo integral del individuo potenciando el conocimiento y desarrollo del lenguaje corporal a través de diferentes técnicas que favorezcan: "revelar o exteriorizar lo más interno y profundo de cada individuo a través del cuerpo y el movimiento, es decir, expresar" Palabras Clave: expresión corporal; multidisciplinario; movimiento; estético; artístico

Abstract: The body language is an important content as a means of communication with their environment. That is, it is a multidisciplinary content of origin, we must define what the concept we left and streams or pillars that support our work in education. We will also define the goals or objectives we want to achieve through the expressive capabilities, sequencing them and showing some didactic concepts for development. The concept of bodily expression has been analyzed from different aspects or areas, as Ortiz says in his text on body language (2002), although this term is ambiguous and versatile, we approached from the educational field, more as an educational tool; yet, never letting go of what conduct or representation communicates all. Ortiz (2002: 25) is an approach to the concept of body language from numerous contributions by various authors. From these states that in the field of physical education and any other material body language should contribute to the development of the individual enhancing the knowledge and development of body language through different techniques favorable "reveal or externalize the innermost and depth of each individual through the body and movement, ie, expressing "reinforce the use of the body and movement as media, ie, communicate and analyze the aesthetic and artistic value of the body".

Key words: body; multidisciplinary; movement; aesthetic; artistic expression 


\section{Introducción}

Cuán importante es considerar los diferentes postulados y diversos autores, para comprender más sobre lo que es la postura corporal, siendo nuestro eje principal, que toda conducta sirve para la comunicación, y para el aprendizaje en cualquiera de sus aspectos. Siguiendo a Ortiz (2002:26) Menciona que la expresión corporal es el medio que utiliza la comunicación cuando se produce a través del cuerpo y el movimiento, desarrollándose un lenguaje propio. Dicho lenguaje puede ser verbal o no verbal, o puede surgir desde la necesidad de la expresión comunicativa, como lo definió la argentina Patricia Stokoe a partir de los años 80, surgiendo la expresión corporal desde la danza y dirigida para personas que carecían de dicho conocimiento, pero que sentían la necesidad desde su organicidad comunicarse corporalmente con los demás, estética o no, se convirtió en un medio de expresión.

Como afirma Motos (1983:11) los hombres en la comunicación interpersonal utilizan básicamente dos sistemas de signos:

- La palabra: como vehículo de la comunicación verbal.

- Y el gesto, la postura, la secuencia de movimientos: estos como canal de la comunicación no verbal.

Cuando nos comunicamos estamos enviando constantemente mensajes corporales a través de la postura, gestos, distancia hacia los demás, vestimenta, etc. Los cuales pueden ser intencionales o no. Continuando nuestro enfoque Ortiz (2002) diferencia entre elementos intencionales o no intencionales del lenguaje corporal.

- Los elementos del lenguaje corporal que se producen sin intencionalidad, de manera inconsciente, involuntaria o espontáneos, y que pertenecen a la comunicación no verbal.

- Los elementos que se producen con intención, consciente, voluntariamente y que pueden ser aprendidos. Estos elementos conformarán la técnica que permitirá desarrollar el potencial expresivo del cuerpo y mejorar sus posibilidades de comunicación.

Sin olvidar que Gardner también describe a esta como la Inteligencia Kinestésica o Corporal dentro de sus famosas "Inteligencias Múltiples" descubiertas en un estudio investigativo en la Universidad de Harvard junto al Dr. Sperry a inicios de los años 60.

Los maestros deben cuidar la comunicación para que el proceso de enseñanza y aprendizaje sea efectivo. Respecto a la comunicación, Jiménez (1991:151) describe cómo en ocasiones los maestros caen en malos hábitos, usando expresiones y actitudes que desconciertan, desvían la atención de los demás y hacen que no los escuchen. Distingue dos factores que bloquean la comunicación: las expresiones verbales, y las conductas no verbales.

Respecto a las expresiones verbales, podemos citar o enunciar: frases y respuestas ásperas, incapacidad de apreciar manifestaciones de buen humor en los demás, hablar al oído a una persona delante de otra, levantar la voz y gritar, hablar en primera persona, no recordar los nombres, cortar a una persona, corregir en público, no mirar al interlocutor, no prestar atención, expresiones vulgares, frases hechas, etc. 
Por otro lado están las conductas no verbales, como por ejemplo: la expresión facial, acciones físicas amenazadoras, nerviosismo, timidez, presunción, suspiros, bostezos o apatía, hábitos como fumar o mascar chicle, transpiración, mostrarse siempre apurado, quejarse continuamente, etc.

Expresiones y conductas de este tipo bloquean las buenas comunicaciones. Un buen maestro debe procurar todo lo contrario, para que la comunicación sea fluida y se de en un clima agradable y positivo. Para evitar estas acciones es necesario realizar un esfuerzo por tomar conciencia de la actuación de uno mismo, analizar cuáles son las expresiones y conductas que individualmente realizamos y obstaculizan nuestra comunicación, y reflexionar sobre las modificaciones que sean necesarias.

La educación es una profesión en la que el dominio del lenguaje corporal es particularmente importante, en gran parte por la influencia que ejerce sobre los jóvenes y su desarrollo. Wainwright (1998:165) plantea un perfil del tipo de conducta no verbal efectiva para un profesor:

"Los profesores deben ser cordiales, cariñosos y gratificantes. Deben ser capaces de llegar a todos los miembros del grupo de estudiantes. Deben estar seguros de sí mismosy bien organizados, y deben ser emocionalmente estables. Es importante prestar atención a las respuestas e intervenciones de los alumnos y evitar ridiculizar o mostrarse sarcástico, hostil, enfadado o arrogante. Deben tener en cuenta las diferencias culturales en el uso del lenguaje corporal".

\section{Desarrollo}

\section{El cuerpo habla}

El cuerpo se comunica siendo el medio de contacto con el entorno, la familia y la cultura. Es decir es una forma comunicativa que se construye con las vivencias como lo mencionan Gubbay y Kalmar (1990), el cuerpo siempre se expresa hasta que fenece, va tomando forma con las experiencias vividas en la familia, la sociedad y la cultura, el individuo interactúa con su medio y de éste recibe información. La misma información que va aportando a la evolución psicosocial, emocional, o forjando personalidades y moldeando estructuras socioculturales en los individuos.

El cuerpo es el lugar de encuentro, el que reconoce y produce vivencias en la interacción con el mundo, creando unos códigos de comunicación y un propio lenguaje. En esta sociedad multicultural llena de acontecimientos provocados por diferentes actores: el estado, la iglesia, los medios de comunicación, la familia, la escuela, la calle, la ciudad, etc. coexisten el cuerpo y su interacción social.

A partir del cuerpo, la expresión se vincula al mundo, a la realidad existente, recreando el contexto y la cotidianidad de forma activa donde no se es un actor pasivo. Es decir, las 
representaciones mentales que se poseen, se manifiestan a través del cuerpo, existe una relación directa de la expresión corporal con lo que se dice, se siente y se hace.

Luego las aulas de clase se convierten en el mágico mundo donde se aprovecha esta concepción para ser desarrollada desde todas las áreas del conocimiento y que permiten al sujeto realizarse y proyectarse en sí mismo de forma auténtica y única. La cotidianidad no existe sin la presencia del cuerpo porque él hace parte de los lenguajes de significación social.

\section{El movimiento elemento esencial:}

Otro de los grandes elementos básicos de la expresión corporal es el movimiento. Como indica el psicólogo Wallon (1987), afirma que el movimiento, es una emoción exteriorizada porque ésta existe de forma inseparable de la motricidad. Determina dos componentes básicos en el movimiento: relación en cuanto al contacto con el mundo exterior, y tonicidad en cuanto a la expresión y relación con el otro, a nivel de relaciones afectivas y cognitivas.

Por eso es aconsejable conocerse a uno mismo para poder entablar empatía con los demás y surta un efecto positivo entre las inteligencias Inter e Intrapersonal de Gardner.

El movimiento diferencia al individuo en su forma de coordinar y regular las funciones motrices, pues en cada persona cambian, afirma que los sujetos se expresan a través de gestos que surgen de sus necesidades de relación. El movimiento no existe sin el contexto de la motricidad, que se enmarca en la capacidad del hombre de desarrollar movimientos por sí mismo.

Así que desarrollar la motricidad en el niño propicia el desarrollo, intelectual, físico y emocional, por esta razón, es esencial que los maestros generen espacios de expresión corporal donde conozcan su cuerpo y el espacio.

El juego es el medio indicado para motivar el movimiento y el lenguaje gestual a través de la imitación que promueve la desinhibición, el ingenio, la imaginación y la iniciativa.

Al reflexionar sobre la expresión corporal y todos sus componentes: el cuerpo, la imaginación, la creatividad, el movimiento, la sensopercepción y la espontaneidad, nos preguntamos cómo se relaciona en una sociedad y desde qué perspectivas se concibe como un ente cultural y social.

\section{Sociología de la expresión corporal:}

Es esencial abordar el tema de la expresión corporal desde la sociología para decir que ésta forma parte de las competencias comunicativas, es la conducta gestual espontánea como lo expresa Hymes (1970), inherente a todo ser humano, es un lenguaje extra verbal, paralingüístico, evidenciado en gestos, actitudes, posturas y movimientos funcionales.

Un lenguaje que se transforma poco a poco, para llegar a la expresión corporal en la toma de un código corporal propio basado en un proceso cinético, que permita la representación y 
creación de imágenes tanto del mundo externo como interno, con un sentido estético comunicativo. La expresión corporal se mira desde la perspectiva de la prosémica y la cinética corporal como esos elementos que nos permiten leer nuestro cuerpo y el del otro.

En ese orden de ideas, la prosémica se relaciona con la comunicación no verbal y que se refiere a la distancia o proximidad física que hay entre los sujetos que mantienen una comunicación. Por lo tanto, es vital decir que ese cuerpo implícito en la relación, desde la cinética usa los movimientos corporales como medio de autoexpresión.

"Una maravillosa armonía enlaza las cosas creadas entre sí, y el ojo del pintor extrae del espectáculo de la vida los elementos de su arte. El batir de las alas de un pájaro nos dará el trazado de un párpado y la ola que muere en la arena, el movimiento de una sonrisa. En el cielo he hallado reflejos aplicables a la mirada, y las flores me han enseñado actitudes para las manos". "Extraigamos las formas de la naturaleza, y las almas de nuestra alma". Leonardo De Vinci.

\section{Metodología}

Para la realización del presente artículo investigativo, con enfoque mixto, aplicamos la Observación Participativa ya que como maestro de expresión corporal, observo los beneficios que mis estudiantes van adquiriendo durante el proceso de enseñanza - aprendizaje, brindándoles seguridad física y emocional a la hora de comunicarse con los demás.

A través del método heurístico y toda la información recabada llegamos a un análisis descriptivo, basándonos en autores y postulados referentes a la necesidad de explorar y aplicar el desarrollo corporal en los educandos como un aporte a su formación holística.

Entre las técnicas usadas tenemos la encuesta que se aplicó a 50 estudiantes de expresión corporal de la Universidad Politécnica del Litoral. Y 50 estudiantes de la Escuela de Arte UEES, ya que nuestra población Universo es de 100 encuestados. El instrumento fue el cuestionario, el mismo que obtenía 10 preguntas con indicador binario. Además se realizó 5 entrevistas a expertos en el área escénica corporal, entre ellos a la PhD Martha Rizzo González (directora de arte uees).

\section{Conclusiones}

El cuerpo del ser humano, es el instrumento de expresión y comunicación por excelencia por lo tanto utiliza como recursos expresivos al gesto y al movimiento. El gesto es necesario para la expresión y la comunicación y el movimiento es la base que permite al niño desarrollar sus capacidades intelectuales, su bienestar físico y emocional.

El 100\% de los encuestados coinciden que la expresión corporal es una actividad que desarrolla la sensibilidad, la imaginación, la creatividad, y la comunicación humana.

Es un lenguaje por medio del cual el individuo puede sentirse, percibirse, conocerse y manifestarse. La Dra. Rizzo concluye diciendo: “La práctica de la expresión corporal 
proporciona un verdadero placer por el descubrimiento del cuerpo en movimiento y la seguridad de su dominio".

El lenguaje corporal permite transmitir nuestros sentimientos, actitudes y sensaciones, el cuerpo utiliza un lenguaje muy directo y claro, más universal que el oral, al que acompaña generalmente para matizar y hacer aquel más comprensible. Todos los otros lenguajes verbal o escrito se desarrollan a partir del lenguaje corporal. Los padres conocen los sentimientos de sus hijos a través de este lenguaje, sabemos cuándo un niño está triste, no es necesario que lo diga, sus gestos y movimientos nos lo indican, su energía disminuye, quizás permanece sentado, con la mirada lánguida, su postura corporal encorvada, los hombros caídos, etc.; son signos que nos sirven para interpretar su estado de ánimo. Todo lo contrario cuando el niño es feliz: saltos, risas, palabras, etc.

Por lo tanto se recomienda considerar el arte de la expresión corporal, en las mallas académicas de educación inicial y básica en pro de un mejor desarrollo físico y emocional de nuestros niños y adolescentes.

\section{Bibliografía}

Arteaga, M. (2003). Fundamentos de la Expresión Corporal. Ámbito pedagógico. Grupo Editorial Universitario. Granada.

Gubbay M., y Kalmar, D. (1990). Expresión Corporal, una manera de danzar; danzar, una manera de vivir.

Guerrero. H. (2014). Desarrollo Creativo, Necesidad imperante en la sociedad ecuatoriana, Danzarte Fundación Cultural. Gamagraf Impresiones, Guayaquil. Ecuador.

Hymes, (1996). From an Underdeveloped Coun- try: Toward Linguistic Competence in The Unites States. En Sociolinguistics, Vol.125. Philadelphia: John Benjamins Publishing Company.

Jiménez, F. (1991). La comunicación interpersonal. Icce. Madrid.

Motos,T. (1983). Iniciación a la expresión corporal. Humanitas. Barcelona

Ortiz, M.M. (2002). Expresión Corporal. Una propuesta didáctica para el profesorado de educación física. Grupo editorial universitario. Granada.

Wainwright, g. (1998). El lenguaje del cuerpo. Pirámide. Madrid.

Wallon, H. (1987). Psicología y educación del niño. Una comprensión dialéctica del desarrollo y la educación infantil. Visor ffi Mec. Madrid 


\section{Anexo:}

\begin{tabular}{|c|c|c|}
\hline Autor & Objetivo & Medio \\
\hline $\begin{array}{c}\text { Ortiz } \\
(2002)\end{array}$ & $\begin{array}{l}\text { Desarrollo integral del individuo } \\
\text { potenciando el conocimiento y } \\
\text { desarrollo del lenguaje corporal }\end{array}$ & $\begin{array}{l}\text { Diferentes técnicas que favorez- } \\
\text { can: } \\
\text { Expresar: exteriorizar lo más in- } \\
\text { terno de cada individuo. } \\
\text { Comunicar: utilizar cuerpo y mo- } \\
\text { vimiento como medio } \\
\text { Analizar el valor estético y artís- } \\
\text { tico del cuerpo. }\end{array}$ \\
\hline
\end{tabular}

\begin{tabular}{|c|l|l|}
\hline Autor & \multicolumn{1}{|c|}{ Objetivo } & \multicolumn{1}{c|}{ Medio } \\
\hline $\begin{array}{c}\text { Arteaga } \\
(2003)\end{array}$ & $\begin{array}{l}\text { Desarrollo potencial de la } \\
\text { capacidad expresiva del ser } \\
\text { humano }\end{array}$ & $\begin{array}{l}\text { Conocimiento personal, comuni- } \\
\text { cación interpersonal, exterioriza- } \\
\text { ción de sentimientos internos del } \\
\text { individuo (gestos, posturas, mo- } \\
\text { vimientos expresivos) }\end{array}$ \\
\hline $\begin{array}{l}\text { Rueda } \\
(2004)\end{array}$ & $\begin{array}{l}\text { Experimentar con las posibilida- } \\
\text { des corporales y la inteligencia } \\
\text { movimiento }\end{array}$ \\
& $\begin{array}{l}\text { emocional. } \\
\text { Transmisión de sentimientos, } \\
\text { pensamientos y actitudes. } \\
\text { Cuerpo como nexo de unión entre } \\
\text { la funcionalidad de la actividad } \\
\text { fisica y la plasticidad de la activi- } \\
\text { dad artistica }\end{array}$ \\
\hline
\end{tabular}

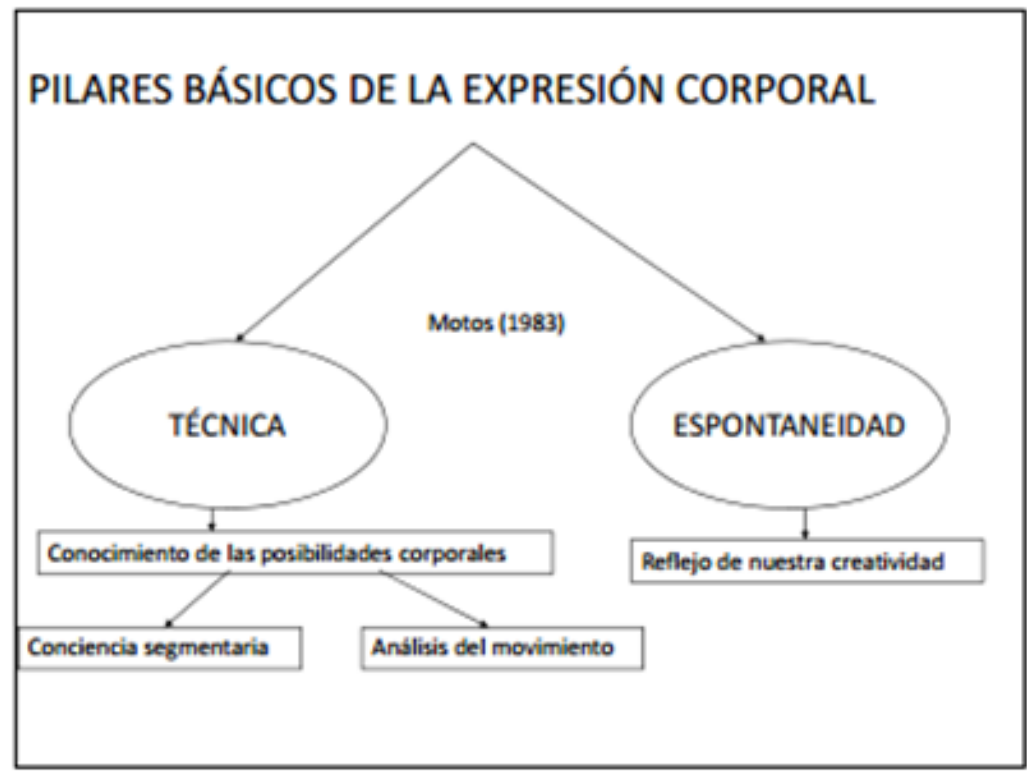

Gráfico n 1.- Pilares básicos de la expresión corporal. 